Running head: VISUAL IMAGERY ON FALSE MEMORIES

This is an Author's Original Manuscript. This article has been accepted for publication (19 Feb 2021) in Memory published by Taylor \& Francis. The final version of the article is available online:

https://www.tandfonline.com/doi/full/10.1080/09658211.2021.1895221.

50 free online copies of the final version of the article are available at https://www.tandfonline.com/eprint/GZSWHQ5IAKF7SCZCTYMJ/full?target=10.108 0/09658211.2021.1895221

Effect of visual imagery on false memories in DRM and Misinformation paradigms

\author{
Frédérique Robin ${ }^{1}$ \\ Emmanuelle Ménétrier ${ }^{2}$ \\ Brice Beffara Bret ${ }^{1}$
}

${ }^{1}$ Laboratoire de Psychologie des Pays de la Loire (LPPL-EA4638), Université de Nantes, Nantes 44000, France

${ }^{2}$ Laboratoire de Psychologie des Pays de la Loire (LPPL-EA4638), Université d'Angers, Angers 49045, France

Correspondence concerning this manuscript should be adressed to: Frédérique ROBIN, Faculté de Psychologie, LPPL - Laboratoire de Psychologie des Pays de la Loire, Université de Nantes, Chemin de la Censive du Tertre, BP 81227, 44312 Nantes Cedex 3, France. EMail: frederique.robin@univ-nantres.fr 


\begin{abstract}
This study is an extension of recent research, which examined the possibility that false memories in the Deese-Roediger-McDermott (DRM) paradigm predict the occurrence of false memories in misinformation paradigm. The purpose was to determine in which extent an imaging instruction reduces false memories in DRM and Misinformation paradigms. A sample of young adults was assigned to the DRM or the misinformation tasks, either in control conditions or in conditions including an imaging instruction. Findings confirm that an imaging instruction decreases false memories in DRM whereas it is not possible to conclude about such a reduction in the misinformation task. Overall, this pilot study suggests that the nature of the stimuli in each paradigm gives rise to quality differences in encoding processes, which in turn have consequences on the monitoring process at retrieval, leading to a weaker misinformation effect than DRM false recognition. In conclusion, while one has argued that the monitoring process is common to both paradigms, false memories in the DRM paradigm would be based on semantic association of words that is, on activation processes in semantic memory, whereas misinformation would rather rely on recollection process in episodic memory. Nevertheless, this hypothesis should be specifically tested in further experiments.
\end{abstract}

(200 words)

Keywords: DRM; false memories; imagery; imaging instruction; misinformation 


\section{Effect of visual imagery on false memories in DRM and Misinformation paradigms}

After numerous demonstrations of false memories in Deese-Roediger-McDermott (DRM, Roediger \& McDermott, 1995) and Misinformation paradigms (Loftus \& Palmer, 1978), the cognitive processes involved in these phenomena are becoming better known. Notably, it was demonstrated that the Activation Monitoring Theory (AMT, Roediger, Balota \& Watson, 2001) or the Fuzzy Trace Theory (FTT, Brainerd \& Reyna, 2005) would be the best explanation of the DRM illusions. In return, the FTT or the Source Monitoring Framework (SMF, Johnson \& Raye, 2000) would be more appropriate for describing false memories in the misinformation paradigm.

Recently, few researchers as James Ost put into question whether one of these theoretical models is more likely to convincingly explain false memories whatever the experimental paradigm (Ost, Blank, Davies, Jones, Lambert, \& Salmon, 2013; Patihis, Frenda, \& Loftus, 2018; Zhu, Chen, Loftus, Lin, \& Dong, 2013). Merits of Ost's work was to point out that the mechanisms underlying false memories seem to be specific to each paradigm.

Based on this finding we may wonder whether the factors that modulate false memories have the same impact whatever the experimental paradigm in which false memories are examined. More precisely, results have shown a reduction of false memories in conditions in which participants encoded DRM lists with the instruction to generate a visual image of object designated by each word (Foley, 2012; Foley et al., 2006; 2009; Robin, 2011; Robin \& Mahé, 2015; Robin, Ménétrier, \& El Haj, 2019). In contrast, studies that have examined the effects of visual imaging on misinformation are still sparse. Therefore, we have to be cautious concerning the hypothesis that imaging encoding would be a good candidate for reducing every sort of false memories of events. Indeed, at the opposite, numerous studies using imaging instruction in false events induction have revealed the negative role of visual images on source recognition with an 
imagination inflation effect or false autobiographical memories (Goff \& Roediger, 1998; Mazzoni \& Memon, 2003; for a review, see Robin, 2010).

In the majority of interviews, eyewitnesses are asked to remember the scene while evoking an image and describing it in detail. However, the presence of vivid and detailed images does not guarantee the veracity of the information collected (whether the images are deliberate or suggested). Hence, the pending issue is to know whether false memories reduction obtained via imagery instructions in the DRM task might be extended to ecological contexts such as eyewitness testimony. Therefore, our aim was to compare the imaging instruction effect in DRM and misinformation paradigms, by using the same kinds of imaging instruction and of memory task (recognition test) in a between-subject design. Participants were randomly assigned to five groups: a control group or a group with imaging instruction during DRM lists encoding for the DRM task or a control group, a group with imaging instructions when presenting misleading information or a group with imaging instructions at the recognition test for the misinformation task,

We expected to replicate previous results showing that imaginal encoding reduces false DRM memories. In the misinformation paradigm, we expected that imaging instruction given during the misinformation questionnaire would amplify the real and plausible nature of the misleading suggestions. This should decrease the amount of correct answers and increase the incorrect answers rates. These performances should be accompanied by a higher feeling of certainty in misleading information. In return, when the imaging instruction was proposed during the recognition task, likely it should reduce the reliability of the suggestions in favor of better discrimination of the source, in similar way to what was generally observed with DRM tasks.

\section{Method}




\section{Participants and design}

Two hundred students $(81 \%$ women $)$, with a mean age of 19.77 years $(S D=1.87)$, voluntarily participated in the experiment. They were first-year psychology students in Nantes and Angers Universities, who were never taught about the topics of the study (i.e., false memories, DRM and misinformation paradigms).

In the DRM paradigm, two conditions were examined in which words were presented orally with an explicit imaging instruction asking participants to generate a visual image of the object corresponding to each word of DRM lists (the imagery condition) or without such instruction (the control condition). The methodology followed was that of the standard DRM paradigm that is an intentional memory condition (Roediger \& McDermott, 1995; Stadler, Roediger, \& McDermott, 1999). Eighty participants were randomly assigned to one of the two conditions of the DRM paradigm. The groups comprised 40 participants both in the control and in the imagery condition (mean age $=20.62 ; S D=2.42$; range: $18-28$ years). All were native speakers of French.

In the misinformation paradigm, three conditions were examined: the control condition (as the one used in Mahé, Corson, Verrier, \& Payoux, 2015) and two imagery conditions (with an explicit visual imaging instruction during the misinformation phase or during the recognition phase). The methodology followed was a French adaptation of Loftus' work including three steps: (1) visualization of an event (a short movie sequence); (2) answering to an unexpected questionnaire containing misleading suggestions about the movie sequence; (3) performing a recognition task about details on the original event (Loftus, Miller, \& Burns, 1978; see, Mahé et al., 2015). One hundred and twenty participants were randomly assigned to one of the three conditions of the Misinformation paradigm. The groups consisted of 40 participants in the control condition and in each of the imagery conditions (mean age = $18.92, S D=1.32$; range was from 17 to 26 years). All were native speakers of French. 


\section{Materials}

The DRM task was composed of 10 lists of eight words strongly associated with a critical lure. These lists were originally developed by Corson, Verrier and Bucic (2009), using the same criteria as the lists of Roediger and McDermott (1995). The words were presented in order of decreasing associative strength to the non-presented related word. The eight highest associates that could be depicted in a visual image were included in the ten lists (see Robin, 2011; Robin \& Mahé, 2015). In previous studies, it was ensured that the imagery value of critical words was high and the same as the imagery value of all the studied words. Each of the study items was presented for 3 seconds. The lists of words were recorded in a female voice and presented to the subjects through headphones. Two presentation orders of lists were used.

A subset of the words presented during the encoding phase was included in the recognition test. This one consisted of 64 words including: 30 of the studied words selected from the presented items in each list (and occurring in the first, third and seventh positions in the lists); 10 non-presented critical words each being a high associate of the words presented in the lists; 24 distractors selected from six other DRM lists that were not presented and unrelated to the studied and critical words. Items on the recognition list appeared in alphabetical order. Every word was rated with a 4-point scale with 1 for "sure it is new", 2 for "probably new", 3 for "probably studied" and 4 for "sure that the item was studied".

The misinformation task consisted of the projection of a short video composed of scenes selected from the film "Z" (Perrin, Rachedi, \& Gavras, 1969) initially tested by Mahé et al. (2015), and which had been first used by Loftus, Levidow, and Duensing (1992). The short video of $6 \mathrm{~min} .19 \mathrm{sec}$ described an assassination attempt of a politician who decided to organize a political rally. The old "Z" film is generally unknown to young participants. 
The misinformation questionnaire used in this research was similar to the one developed by Mahé et al. (2015), according to Loftus' studies (Loftus et al., 1978, 1992). Twelve open questions on the video sequence were presented in chronological order. Most of them concerned the visual aspects of the events. Four leading questions suggested consistent information (e.g., [Question 10]. At the end of the film, when everyone is gathered in the public square, a blue vehicle arrives at high speed. What type of vehicle is it?). Four misleading questions suggested inaccurate information (e.g., [Question 10]. At the end of the film, when everyone is gathered in the public square, a white vehicle arrives at high speed. What type of vehicle is it?). Four neutral questions suggested no information concerning the event (e.g., [Question 10]. At the end of the film, when everyone is gathered in the public square, a vehicle arrives at high speed. What type of vehicle is it?"). There were three versions of the questionnaire in order that every question was presented the same number of times in a leading, misleading, or neutral way across participants. The question order effect was controlled with no successive question of the same type following each other (leading, misleading or neutral). The number of participants was similar for each version of the questionnaire.

The recognition test was constituted of 12 questions relying on the leading, misleading and neutral information to which the participants were exposed during the misinformation phase. The questionnaire administered consisted in a two-alternative forced choice task, for which the participants had to choose between two answers referring to the leading and misleading information (e.g., [Question 10] At the end of the film, what color is the vehicle arriving at high speed? Proposed answers: Blue or White). For each question, participants had to indicate their confidence rating on a five-points Likert scale (from not sure at all to completely sure). All participants received the same version of the recognition test, in which the questions were presented according to the chronological order of events occurring in the movie. The correct response was systematically the first of the two proposed alternatives. 
Moreover, two additional questions allowed to ensure that participants had not seen the film before, which was the case for each of them, and had not understood the objectives of the experiment.

\section{Procedure}

In the DRM task, participants completed the experiment individually. Following their consent, they received the study instructions. In the control condition, participants were simply instructed to pay careful attention to each word in order to carry out another task later on these words. In the imaginal condition, participants were instructed to imagine each object depicting the word and to rate the vividness of their image on a 1-7 scale ("no image" to "an image as vivid as real vision"). After studying the 10 lists, all participants performed the incident recognition test and then completed the VVIQ.

In the misinformation task, participants were divided in three conditions. The experimentation comprised three phases and had been done during a 1st year university work methodology course. In each condition, the 40 participants watched the movie all together. They were only instructed to pay full attention to this event. One hour and a half after having seen the event, without participants being warned, the experimenter came back in the course and students were invited to answer the 12 open questions of the misinformation questionnaire. Then after having answered to these questions, they were told, this time the experiment was closed. Nevertheless, one week later, the participants had to perform the forced-choice recognition test about the movie sequence they watched before during the same course. Participants had to choose between the right (leading question) or the false answers (misleading question). For each answer, they estimated their feeling of confidence on a five-points scale (from not sure at all [1] to very sure [5]). They were asked to answer to all questions. In the three conditions, the procedure was exactly the same, excepted that in the imaging- 
misinformation or imaging-recognition conditions, participants were instructed to imagine the scene described in each question as vividly as possible (i.e., as if the scene were unfolding before their eyes) during the misinformation or recognition phases, respectively. After performing the recognition test, all participants completed the VVIQ. Then, all participants received debriefing about experiment's aims.

\section{Data analyses}

The analyses were conducted to ensure that false memories in the DRM task as well as a misinformation effect were observed; to determine whether there was an effect of imaging instruction in both of these tasks and if so, what the pattern of this effect was. We performed conventional (metric and frequentist) analyses on all of measures of interest in order to be able to compare our results to previous studies on the topic. We also applied multilevel Bayesian ordinal analyses on our main outcome (false memories) in order to i) increase the number of observations used in the analyses, ii) fit more precise models (e.g. see Bürkner \& Vuorre, 2019; Liddell \& Kruschke, 2018) and iii) model varying effects for both participants and items.

\section{Results}

\section{DRM and imagery effect}

\section{Frequentist analyses}

The mean proportions of "old" responses (i.e., responses 3 and 4 of the rating scale) associated to each item type are shown in Table 1. These answers correspond to items identified as previously studied, and thus refer to correctly recognized studied items; false recognitions of critical lures (non-studied, associated with the studied words); and false recognitions of distractors (non-studied, not associated with the studied and critical words), respectively. 
Table 1. Mean proportion (standard deviation) of « old» responses (3-4) for each item type (studied; lures; distractors) in each experimental condition (control vs imagery)

\begin{tabular}{llll}
\hline & Studied words & Lures & Distractors \\
\hline Control & $.81(.11)$ & $.54(.28)$ & $.08(.07)$ \\
\hline Imagery & $.91(.07)$ & $.35(.24)$ & $.03(.04)$ \\
\hline
\end{tabular}

An ANOVA with repeated measures was carried out with Item type as a within-subject factor (studied words, lures and distractors) and Condition as a between-subject factor (control vs imagery). The analyses revealed a significant effect of Condition, $F(1,78)=5.11, p=.02$, $n_{p}^{2}=.06$; a significant effect of Item, $F(2,156)=478.47, p<.001, n^{2}{ }_{p}=.86$, which indicated the presence of false memories in the DRM paradigm. Indeed, post-hoc analyses (Bonferroni) indicated that rates of veridical recognition for the studied words $(M=.86 ; S D=.11)$ were significantly higher than false recognitions of lures $(M=.45 ; S D=.28), t(78)=15.92, p<.001$, Cohen's $d=3.461$. False recognitions of lures were higher than false recognitions of distractors $(M=.05 ; S D=.06), t(78)=15.02, p<.001$, Cohen's $d=1.680$. The analyses also reported a significant Condition $\mathrm{x}$ Item interaction effect, with $F(2,156)=16.03, p<.001, n^{2} p$ $=.17$. Post-hoc analyses (Bonferroni) showed that correct recognitions rates of studied words were slightly higher in the imagery than in the control condition, $t(78)=2.81, p=.07$ (see Table 1). In return, false recognitions of distractors were the lowest and of equivalent proportion in both imagery and control conditions, $p=1.000$. Then, false recognitions of lures were significantly reduced in the imagery compared to the control condition, $t(78)=5.20, p<.001$. 
To further investigate the effect of imagery on DRM false memories, the mean confidence ratings were compared for the two conditions (control vs imagery) on the 4-point scale. Table 2 presents the mean rating confidence for each encoding condition and each item type. The analyses revealed a significant effect of Condition, $F(1,78)=9.77, p=.002, n^{2} p$ $=.11$; a significant effect of Item, $F(2,156)=422.29, p<.001, n^{2}{ }_{p}=.84$. Post-hoc analyses (Bonferroni) indicated that confidence rates for the studied words were significantly higher than false recognitions of lures, $t(78)=11.46, p<.001$, Cohen's $d=1.280$. Confidence rates for lures were higher than confidence for distractors, $t(78)=12.14, p<.001$, Cohen's $d=1.358$. The analyses also reported a significant Condition x Item interaction effect, with $F(2,156)=13.98$, $p<.001, n_{p}^{2}=.15$. Post-hoc analyses (Bonferroni) showed that confidence rates for studied words were equivalent in the two conditions, $t(78)=2.40, p=.25$ (see Table 2). In return, false recognitions of distractors were lower in the imagery condition, $t(78)=2.98, p=.05$. Then, false confidence of lures were significantly reduced in the imagery compared to the control condition, $t(78)=4.81, p<.001$

Table 2. Mean confidence ratings (standard deviation) on 4-point scale for each item type (studied; lures; distractors) in each experimental condition (control vs imagery)

\begin{tabular}{llll}
\hline & Studied words & Lures & Distractors \\
\hline Control & $3.41(.03)$ & $2.61(.08)$ & $1.50(.03)$ \\
\hline Imagery & $3.66(.03)$ & $2.10(.06)$ & $1.18(.02)$ \\
\hline
\end{tabular}

\section{Bayesian analyses}

A multilevel Bayesian ordinal regression was fitted with Item type as a within-subject factor (studied words, lures and distractors, respectively coded as a linear contrast 1, 0 and -1) 
and Condition as a between-subject factor (control vs imagery, respectively coded 0.5 and -0.5 ) as well as their interaction as fixed factors and varying intercepts for participants and items (words) in the model. The dependent variable was the response of the participants $(1,2,3$, or 4). Data analysis was performed with the brms R package (Bürkner, 2018). Dispersion of each participant's responses as a function of item type and experimental condition is depicted in Figure 1.

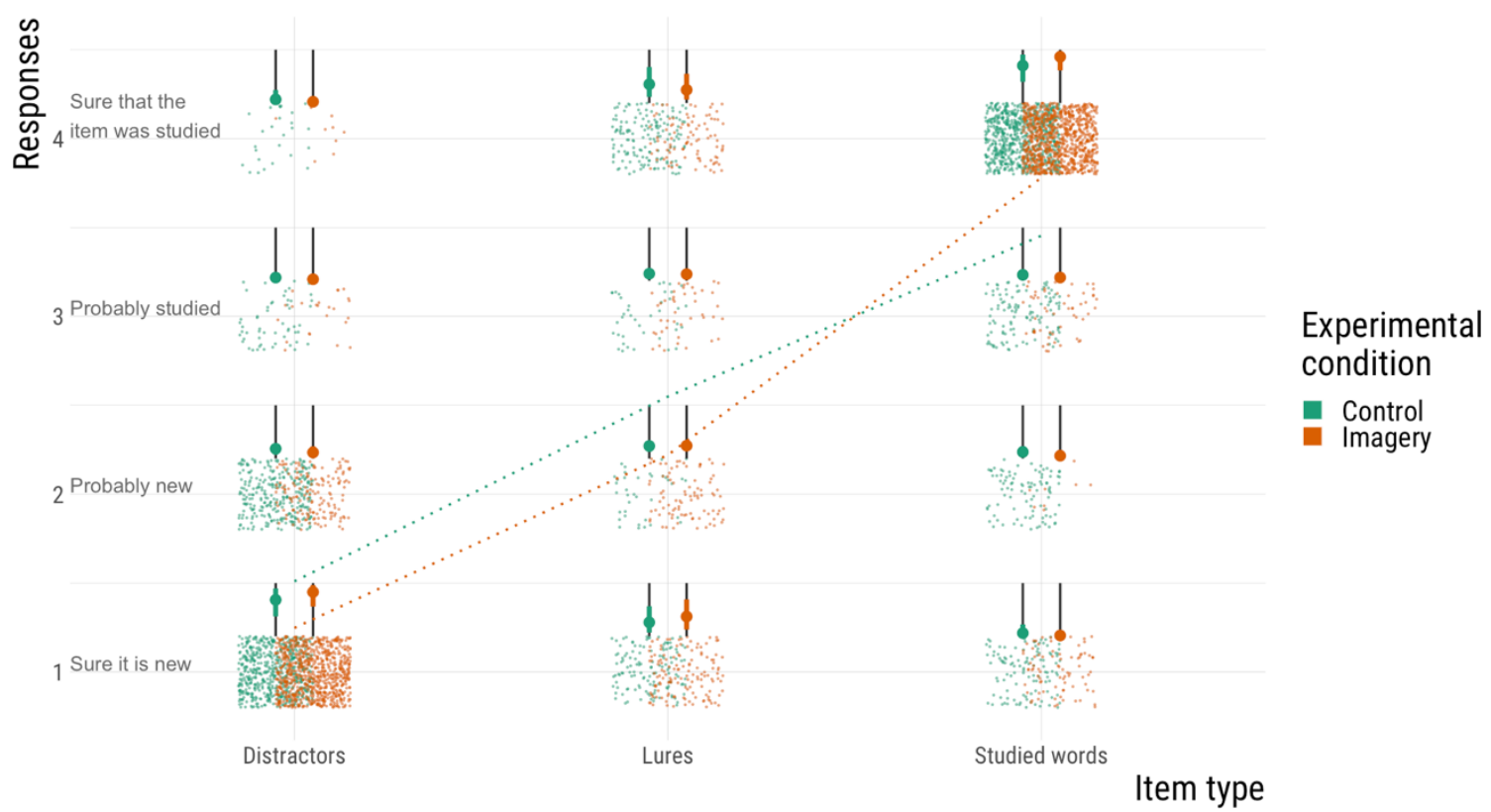

Figure 1. Responses as a function of item type and experimental condition.

In Figure 1, each small dot represents one response of a participant (the position of each dot was slightly modified for readability). Larger dots above represent estimates of responses probability and are presented along with their 95\% CI (credibility interval) as estimated by the model. Dashed lines link the sum of the responses x their probability as estimated by the model.

The analyses did not allow to conclude about an (absence of) effect of Condition, $\beta=$ $0.03,95 \%$ CI $[-0.13,0.19]$, but a substantial effect of Item type, $\beta=1.28,95 \%$ CI $[1.18,1.37]$ was found, indicating that rates of veridical recognition for the studied words were higher than 
false recognitions of lures, which were higher than false recognitions of distractors. When the item type variable was coded as an orthogonal contrast $(-1,2,-1)$, we could not conclude about an (absence of) effect of this variable, $\beta=-0.04,95 \% \mathrm{CI}[-0.32,0.25]$, suggesting that the linear contrast was the best model. The analyses also reported a substantial Condition $\mathrm{x}$ Item type interaction effect, $\beta=-0.52,95 \%$ CI $[-0.60,-0.44]$, suggesting that the effect of imagery (vs. control condition) on accuracy was more important for higher values of the linear contrast (see Figure 1).

These results replicated those found in previous studies showing a constant reduction in false memories with an imaging instruction (Burns, Jenkins, \& Dean, 2007, exp.2; Foley, 2012; Foley Wozniak, \& Gillum, 2006; Gunter, Bodner, \& Azad, 2007; Olszewska \& Ulatowska, 2013; Perez-Mata, Read, \& Diges, 2002; Robin \& Mahé, 2015; Robin et al., 2019).

\section{Misinformation and imagery effect}

As a reminder of our hypotheses, in the control condition, the misinformation effect should be reflected in the difference of correct answers between suggestive conditions, that is, with a higher rate of correct responses in leading or neutral conditions than in misleading condition (see Mahé et al., 2015). Nevertheless, compared to the control condition, the imaging instruction should increase the misinformation effect when participants were encouraged to imagine false information during the misinformation questionnaire. At the opposite, imaging instruction should reduce the misinformation effect when participants were invited to visualize the scene for each question of the recognition test. To sum up, false memories rates should be lower in the imagery-recognition condition than in the control one. In return, false memories should be higher in the imagery-misinformation condition than in the control condition.

\section{Frequentist analyses}


The mean proportions of correct answers collected for each question type as a function of experimental condition are shown in Table 3.

Table 3. Mean proportions (standard deviation) of correct answers for each Question type (leading, neutral, misleading) in each experimental condition (control; imagerymisinformation; imagery-recognition)

\begin{tabular}{lcll}
\hline & Leading question & Neutral question & Misleading question \\
\hline Control & $.89(.16)$ & $.85(.19)$ & $.64(.18)$ \\
Imagery-misinformation & $.84(.18)$ & $.82(.16)$ & $.69(.21)$ \\
Imagery-recognition & $.85(.21)$ & $.84(.18)$ & $.67(.26)$ \\
\hline
\end{tabular}

An ANOVA with repeated measures was carried out with Question type as a withinsubject factor (leading, misleading, neutral) and Condition as a between-subject factor (control, imagery-misinformation, imagery-recognition). The analyses were conducted on the mean proportions of correct responses (correct and incorrect answers being complementary in the forced-choice recognition test). The analyses revealed a significant effect of Question type, $F(2,234)=37.47, p<.001, n_{p}^{2}=.24$. Post-hoc analyses (Bonferroni) showed that correct answers reached the lowest rates in the misleading $(M=.67 ; S D=.22)$ condition compared to the neutral $(M=.84 ; S D=.18)$ and leading $(M=.86 ; S D=.18)$ conditions, all $p s<.001$. Rates of correct answers were high and of the same magnitude in the neutral and leading conditions, $p=1.000$. These results replicated the misinformation effect reported in previous studies (see Mahé et al., 2015). However, the analyses did not allow to conclude about an (absence of) effect of Condition effect, $F(2,117)=0.05, p=.94$, nor about an (absence of) Condition $\mathrm{x}$ Question type interaction effect, $F(4,234)=0.98, p=.41$. Hence, it is possible to conclude that imaging 
instruction reduce or increase false memories in the misinformation paradigm whereas it reduced significantly false memories in the DRM paradigm.

\section{Bayesian analyses}

A multilevel Bayesian logistic regression was fitted with Question type as a withinsubject factor (leading, misleading, neutral, respectively coded as a quadratic contrast $1,-2$ and 1) and Condition as a between-subject factor (control, imagery-misinformation, imageryrecognition, respectively coded as a linear contrast $0,-1$ and 1) as well as their interaction as fixed factors and varying intercepts for participants and items (words) in the model. The dependent variable was the response of the participants ( 0 for incorrect or 1 for correct answers, correct and incorrect answers being complementary in the force-choice recognition test). Data analysis was performed with the brms $\mathrm{R}$ package (Bürkner, 2018). Dispersion of each participant's responses as a function of item type and experimental condition is depicted in Figure 2.

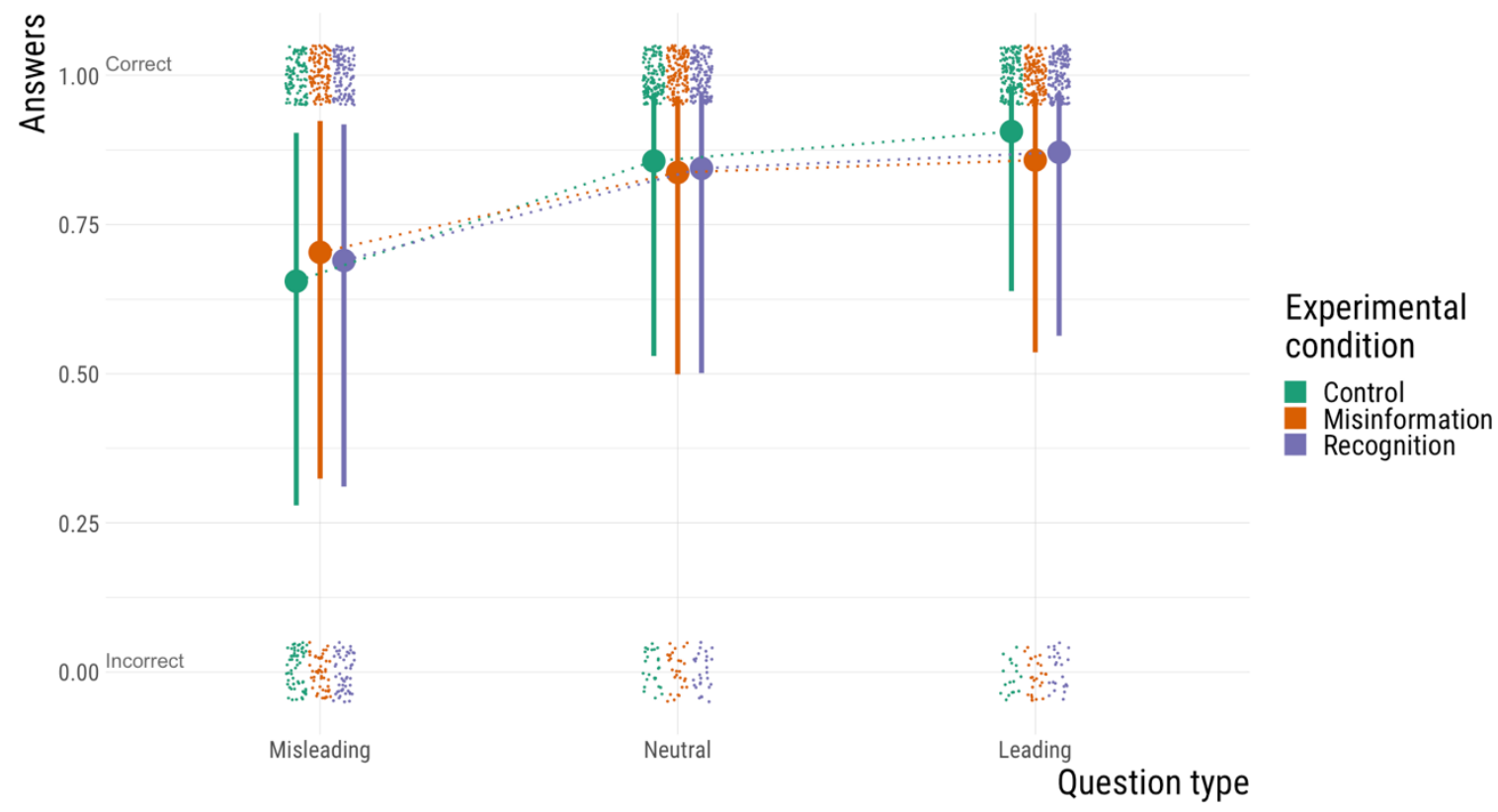

Figure 2. Answers as a function of item type and experimental condition. 
Each small dot represents one answer of a participant (the position of each dot is slightly modified for readability). Larger dots in the middle represent median estimates of responses probability and are presented along with their $95 \% \mathrm{CI}$ as estimated by the model.

The analyses revealed a substantial effect of Question type, $\beta=0.35,95 \%$ CI $[0.00$, 0.69]. Correct answers reached the lowest rates in the misleading condition compared to the neutral and leading conditions. When the question type variable was coded as a linear contrast $(1,0,-1)$, there it was not possible to conclude about an (absence of) effect of this variable, $\beta=$ $0.14,95 \%$ CI $[-0.61,0.90]$, suggesting that the quadratic contrast was the best model. The analyses did not allow to conclude about a (absence of) Condition effect, $\beta=0.01,95 \%$ CI [$0.16,0.19]$, nor a Condition $\mathrm{x}$ Question type interaction effect, $\beta=0.02,95 \%$ CI $[-0.09,0.13]$. This was also true when the question type variable and the condition variable were coded as orthogonal contrasts (linear and quadratic respectively), with a $\beta=0.02,95 \% \mathrm{CI}[-0.09,0.12]$ for the Condition effect and a $\beta=0.04,95 \%$ CI $[-0.09,0.17]$ for the interaction effect.

\subsection{Confidence in the answers}

During the recognition test, participants estimated their level of confidence on a fivepoints scale (from not sure at all [1] to very sure [5]). Mean rates of confidence level reported in correct answers are shown in Table 4. An ANOVA with repeated measures was carried out with Question type as a within-subject factor (leading, misleading, neutral) and Condition as a between-subject factor (control, imagery-misinformation, imagery-recognition). Analyses computed on each participants' mean confidence ratings (correct answers) revealed neither an effect of Question type, $F(2,230)=.44, p=.64$, nor of Condition, $F(2,115)=.48, p=.61$. Moreover, the interaction between these two variables was not significant, $F(4,230)=.58, p$ $=.67$. 
Table 4. Mean confidence rates (standard deviation) for each question type and each condition in misinformation paradigm

\begin{tabular}{lcll}
\hline & Leading question & Neutral question & Misleading question \\
\hline All conditions & $3.87(0.81)$ & $3.79(0.74)$ & $3.79(0.99)$ \\
\hline Control & $3.85(0.83)$ & $3.79(0.79)$ & $3.78(1.01)$ \\
Imagery-misinformation & $4.04(0.75)$ & $3.87(0.69)$ & $3.76(1.05)$ \\
Imagery-recognition & $3.70(0.84)$ & $3.71(0.74)$ & $3.82(0.93)$ \\
\hline
\end{tabular}

\subsection{Relationships between correct answers and confidence rates}

To determine whether there was a relationship between giving correct answers at the recognition test and confidence rate, Pearson's correlation coefficients were calculated between the total confidence rates scores observed for each participant and the number of corresponding correct answers for each question type (leading, misleading and neutral). Correlation coefficients are shown in Table 5. Correlations were significant, indicating that the higher the number of correct answers, the more confident the participants were in their answers whatever the question type. This analysis was run by eliminating participants with missing values $(N=$ 117).

Table 5. Pearson's correlation coefficients between total confidence score for correct answers as a function of question type (leading, misleading and neutral).

\begin{tabular}{llll}
\hline & Number of correct answers for each \\
& question type & \\
\hline Confidence Correct question & $\mathbf{. 6 6 2} * * *$ & ------ & ------
\end{tabular}


Confidence Neutral question

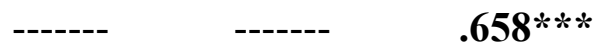

Confidence Misleading question

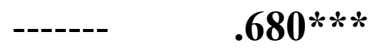

Notes: $N=117 ; * * * p<.001$

\section{Imagery ability check}

To ensure that there was no bias due to individuals' imagery ability, we considered the effect of individual imagery capacities on the basis of the Vividness of Visual Images Questionnaire (VVIQ, Marks, 1973). The analyses carried out on scores for the VVIQ as a function of experimental condition in the DRM task were not significant, $t(78)=1.19, p=.23$; all participants had relatively moderate VVIQ scores (see Table 5). This imagery propensity for all participants is a new element corroborating the imaging instruction effect on the reduction of false memories in the DRM paradigm. Therefore, reduction in false memories observed in the DRM task results from imagery coding during encoding and not from natural higher imagery abilities in the imagery group compared to the control group.

In the same vein, all participants in the misinformation condition had relatively moderate imagery abilities. The analyses of VVIQ scores as a function of experimental condition were not significant (see, Table 5). Nevertheless, the failure to find an effect of imagery ability on misinformation false memories such as it was also the case for DRM false memories underlines the VVIQ limits.

Table 5. Mean scores (standard deviation) of VVIQ in each condition of the DRM and misinformation paradigms 


$$
\begin{aligned}
& \text { Control } 61.75(8.42) \\
& \text { Imagery } 59.35(9.42) \quad t(78)=1.19, p=.23
\end{aligned}
$$

Misinformation paradigm

$$
\text { Control } 59.35(8.03)
$$

Imagery-misinformation $57.47(9.55)$

Imagery-Recognition $58.97(8.96) \quad F(2,117)=0.50, p=.61$

\begin{abstract}
Discussion
The objective of this study was to examine the effect of an imaging instruction on false memories in the DRM and misinformation paradigms. Specifically, we expected to replicate previous results showing that imaging instructions during encoding reduced false DRM memories. In the misinformation paradigm, we suggested that imaging instruction given during the misinformation questionnaire should amplify the real and plausible nature of the misleading suggestions (i.e., decrease the rates of correct responses and increase rates of incorrect responses). On the other hand, the imaging instruction given during the recognition task should reduce the reliability of the suggestions for better source discrimination as it was previously observed in DRM tasks. Therefore, the main question was whether, under certain circumstances, the imaging instruction could reduce false memories in both paradigms. This question was intended to provide further support in favor of the similarity or differences between the two paradigms, as a few past findings have shown that they may rely on different mechanisms (Monds, Paterson, \& Kemp, 2016; Ost et al., 2013; Patihis et al., 2018; Zhu et al., 2013).
\end{abstract}

Our findings revealed false memories effect in DRM and misinformation tasks replicating previous studies (Roediger \& McDermott, 1995; Loftus et al., 1978). We found that 
imaging instruction reduced DRM false memories in line with past findings (Foley, 2012; 2009; 2006; Gunter et al., 2007; Robin, Ménétrier, \& El Haj, 2019; Robin \& Mahé, 2015; Robin, $2011 ; 2010)$. In contrast, it was not possible to conclude that the imaging instruction decreases or increases false memories in the misinformation task. Although our results were obtained with between-subject design, they were in accordance with within-subject design past studies, as one conducted by Ost et al., (2013). In addition, Wilkinson \& Hyman (1998; Qin, Ogle, \& Goodman, 2008) found that false memories in the DRM did not correlate with distortions of imagined autobiographical events.

The imagery effect on DRM lists could be explained in two ways. According to the Activation Monitoring Theory (McDermott \& Watson, 2001; Roediger, Watson, McDermott, \& Gallo, 2001), imaging instruction leads participants to focus on the visual characteristics of the objects corresponding to the studied words that are depicted in their visual images. Hence, the imaging instruction focused attention on studied words and reduced the activation of associated words. At retrieval, visual images enabled a reliable source monitoring based on visual characteristics whose lures were not provided. The retrieval of these distinctive details (i.e., visual images) allowed participants to accept an item as previously seen or heard. Alternatively, conforming to the distinctiveness heuristic hypothesis (Dodson \& Schacter, 2001; 2002; Israel \& Schacter, 1997; Schacter, Israel, \& Racine, 1999), visual images built during encoding help monitor cues during the retrieval test to distinguish lures from the studied words. This decision strategy would be based on a strict criterion using distinctive characteristics of the encoding condition. Overall, these theoretical explanations consider that building visual images at encoding becomes a criterion of decision at the time of recovery, with the lures not provided with images being rejected (Foley, 2012; Gunter et al., 2007; Robin \& Mahé, 2015). 
Now, how to explain the lack of imagery effect in misinformation task? These null findings must be interpreted with caution; nevertheless, they could have several potential explanations that create new research perspectives. One explanation could provide from the nature of stimuli used in the misinformation task and a fortiori result from difference in encoding processes between DRM and misinformation tasks. Indeed, relying on the dual coding theory (Paivio, 1973; 1991), we may hypothesize that the video footage as audio-visual stimulus, automatically and implicitly, has elicited perceptual processing leading to elaboration of visual images. Dual coding theory (Paivio, 1986; 2007) assumes that imaging code plus verbal code improved memory performance and reduced interference. In the misinformation task, the video footage automatically induces an imaginal coding plus a verbal coding. Hence, in the control condition of the misinformation paradigm, correct memories seem to be higher and false memories lower than in DRM control condition. Although results providing from recognition test in DRM and misinformation tasks are not statistically comparable, they tend to corroborate this hypothesis. Indeed, in control conditions, false memories rates are lower in the misinformation task $(M=.36)$ than in the DRM one $(M=.54)$, the latter implying implicitly and automatically a verbal encoding only. As Paivio suggested (2007), imaginal code for words relies on intentional processes due to a lower probability of evocation of a visual image for concrete words relative to drawings. Hence, dual coding for visual materials is more efficient than for verbal materials. However, false memories rates reached the same magnitude when participants in DRM intentionally added to the verbal coding, of word lists, an imaging coding via an explicit imagery instruction $(M=.35)$. In the DRM imagery-condition, participants performed a dual coding (verbal plus imaginal).

An alternative explanation is that visual images corresponding to the video footage would provide a sufficiently distinctive cue to cause less misattribution between original event and suggested events, without to preclude the creation of false memories. This interpretation 
should be confronted to the past studies having shown a reduction of DRM false memories in which words were presented with corresponding drawing. Indeed, it was suggested that drawings processing gave rise to visual images reducing false memories. Schacter and Israel (1997; Norman \& Schacter, 1997) assumed that false recognition was reduced by creating conditions favoring the encoding of perceptual details distinctive to each item. Therefore, the heuristic of distinctiveness that explains the reduction of false memories in the DRM could also explain the implicit imagery effect in misinformation paradigm and hence our null effects of explicit imagery instruction, imaging coding being automatic. In this view, source monitoring would be more effective in the misinformation task whereas successful monitoring in DRM would depend more on contextual support provided during the encoding like pictures or explicit imaging instruction (Foley, 2012; Robin \& Mahé, 2015; Schacter \& Israel, 1997). The distinctive characteristics of the perceived events (e.g. video footage; drawings; visual images) enables to distinguish them from activated or inferred memories, thus favoring the process of discrimination during the recovery phase. Therefore, we may assume that it is the nature of the encoding applied to the events that contributes to reduce false memories.

In DRM tasks, the semantic networks activation and verbal coding lead to a semantic representation poor in distinctive details useful for an efficient source monitoring. In misinformation, visual and verbal encoding lead to an episodic representation rich in distinctive details, increasing source monitoring efficiency (Zhu et al., 2013). In this view, creation of false memories in each paradigm likely hinges on different encoding processes. Therefore, false memories in DRM may not predict memory distortions in the misinformation task. These conclusions are in line with James Ost's work and past studies that found no reliable relationship between false memories in these both paradigms (Falzarano \& Siedlecki, 2019; Nichols \& Loftus, 2019; Patihis et al., 2018; Ost et al., 2013; Zhu et al., 2013). 
Although our study was purely exploratory, it may be considered as an extension of James Ost's conception about false memories paradigms, for who the only common mechanism between both paradigms would be the monitoring process. Indeed, as he suggested, both types of false memories "could also [...] be seen as source monitoring failures" (Ost et al., 2013, p.5). James Ost and his colleagues questioned the concept of false memories by showing that individuals prone to false DRM memories were not just as likely to produce effects of misinformation. It was the first study on this issue. These authors compared false memories in within-subject design with five DRM word lists and a short video footage on recall and recognition tests. Although false memories rates were in the same magnitude, they were not correlated as well as sensitivity measured with signal detection indices. Ost et al. (2013) have discarded several explanations like unreliable measurement between recognition in each paradigm, difference in the samples or lack of power. We may add that neither the contamination bias between tasks performed by the same participants, nor the cognitive load resulting from successive tasks explained these differences. Although, in our study, these potential effects were controlled with between-subject design, the imaging instruction led to mixed results: a significant effect in DRM and no significant effect in misinformation. In accordance with James Ost et al. suggestions (2013, p.5), DRM false memories could be classified as "naturally occurring" and referring to a "normal associative and reconstructive process". Indeed, false memories in DRM result from an internal and implicit activation during the encoding and from a failure of source monitoring increased by the presence of lures in the recognition test. These lures automatically reinforce semantic associations with studied words, and hence endorse the likelihood to judge that they have been encountered before. In contrast, misinformation effect will be classified as "suggestion dependent" resulting from an external and explicit misleading suggestion (post encoding event). Nevertheless, differences in encoding processing between both paradigms discussed before, question the robustness of false memories 
based on implicit activation mechanisms (DRM task) compared to suggestions prompting a recollection of events and based on explicit mechanisms. To conclude, both kinds of false memories may hinge on different encoding processes. Therefore, we must be particularly attentive, as James Ost suggested, when we seek to identify memory errors like false memories. Until proven otherwise, it should be clarified whether it is a DRM false memory or a misinformation effect.

Several limitations of the present study need to be mentioned. First, we compared the imaging instruction effect with a between-subject design, which has precluded the analyses about relationships between both paradigms. We did not choose within-subject design because it requires a larger number of participants and it was not the objective of this pilot study. Moreover, a within-subject design would have likely increased the cognitive load, participants having to perform several successive memory tasks. In addition, it would be likely that the imaging instruction included in a task would have amplified its effect in a subsequent task. This restriction has limited the potential analyses. Second, our current sample size did not allow to detect our effect of interest with a satisfying power. Indeed, interaction effects require larger sample sizes in order to be tested, even when targeting medium to large effect sizes. Nevertheless, the current pilot study was crucial for setting the statistical models by shedding light on the patterns of data in these paradigms. Consequently, we will replicate this exploratory pilot work in future research. Our objective is i) to cross-check the data from two paradigms carried out independently in order to shed light on the processes involved in false memories first and ii) underline the methodological limits with a view to a more rigorous study. The aim is therefore to collect more data in the future with clean power analysis thanks to simulations based on the models we fitted in this study. We will also perform sequential analysis in order to optimize our sample size. Third, we used in each task different formats for the encoding and retrieval phases. Like in standard procedure, misinformation effect consisted to see a video 
footage whereas DRM word lists were presented orally. The formats of recognition tests also varied: a forced-test choice and a confident scale on 5 points in misinformation task, while DRM used confident scale on 4 points. Future studies should distinguish between yes/no responses and confident scales in order that, all other things being equal, both paradigms should be comparable. Indeed, we want to underline that a significant result in a paradigm and a nonsignificant result in the other is not statistically sufficient (nor necessary) to conclude that the difference between the two paradigms is significant. We will need to make the paradigms statistically comparable in order to specifically test the difference between the two.

Considering these limitations, the results of this pilot study should be taken with caution. Further research is needed to compare more rigorously, on a larger sample, the effect of visual vs. verbal information on false memories in these paradigms. Notably, with a within-subject design, it will be interesting to compare combined DRM picture-word lists with video footage misinformation. Dual coding (visual images plus verbal) does affect false memories in similar way in both paradigms.

\section{Conclusion}

In line to James Ost's works and his colleagues (DePrince, Allard, Oh, \& Freyd, 2004; Pezdek \& Lam, 2007), we may conclude that false memories refer to novel events that have never been experienced before (more often traumatic events like childhood sexual abuse), which are completely different from word lists memory errors. Misinformation paradigm remains likely closer to natural situations of testimonies, which are mostly based on visual and auditory details. Nevertheless, DRM may be considered as an interesting tool for the exploration of memory illusion, which is mnemonic dysfunctions in neurologic or psychiatric disorders (e.g. Korsakoff syndrom; schizophrenia; Alzheimer disease).

(6588 words including Tables and Figures) 


\section{Compliance with Ethical Standards}

The data has not been previously published, and it is not under simultaneous consideration by another journal. Research ethics were applied in accord with the APA ethical standards. My coauthors and I do not have any interests that might be interpreted as influencing the research. Participants were fully informed of the current research, freely consented to participate and were able to withdraw whenever they wished.

\section{Open Practices}

Data (for frequentist and Bayesian analyses) and scripts for (Bayesian) analyses have been made publicly available via the Open Science Framework and can be accessed at $\underline{\text { https://osf.io/zsh3b/. }}$

\section{References}

Brainerd, C. J., \& Reyna, V. F. (2005). Oxford psychology series. The science of false memory. Oxford University Press.

https://doi.org/10.1093/acprof:oso/9780195154054.001.0001

Bürkner, P.-C. (2018). Advanced Bayesian Multilevel Modeling with the R Package brms. The R Journal, 10(1), 395. https://doi.org/10.32614/RJ-2018-017

Bürkner, P.-C., \& Vuorre, M. (2019). Ordinal Regression Models in Psychology: A Tutorial. Advances in Methods and Practices in Psychological Science, 2(1), 77-101. https://doi.org/10.1177/2515245918823199

Burns, D., Jenkins, C. L., \& Dean, E. E. (2007). Falsely recalled items are rich in itemspecific information. Memory \& Cognition, 207, 1630-1640.

http://dx.doi.org/10.3758/BF03193497 
Corson, Y., Verrier, N., \& Bucic, A. (2009). False memories and individual variations: The role of Field Dependence-Independence. Personality and Individual Differences, 47, 1, 811. http://dx.doi.org/10.1016/j.paid.2009.01.036

De Prince, A. P., Allard, C. B., Oh, H., \& Freyd, J. J. (2004). What's in a name for memory errors? Implications and ethical issues arising from the use of the term "false memory" for errors in memory for details. Ethics \& Behavior, 14,201-233.

http://dx.doi.org/10.1207/s15327019eb1403_1

Dodson, C. S., \& Schacter, D. L. (2001). "If I had said it I would have remembered it": Reducing false memories with a distinctiveness-heuristic. Psychonomic Bulletin \& Review, 8, 155-161. http://dx.doi.org/10.3758/BF03196152

Dodson, C.S., \& Schacter, D.L. (2002). When false recognition meets metacognition: The distinctiveness heuristic. Journal of Memory and Language, 46, 782-803. https://doi.org/10.1037/e501882009-058

Falzarano, F., \& Siedlecki, K. L. (2019). Investigating the relations among different measures of false memory. Advances in Cognitive Psychology, 15(4), 290-300. https://doi.org/10.5709/acp-0276-0

Foley, M. A., Hughes, K., Librot, H., \& Paysnick, A. (2009). Imagery encoding effects on memory in the DRM paradigm: A test of competing predictions. Applied Cognitive Psychology, 23(6), 828-848. https://doi.org/10.1002/acp.1516

Foley, M. A., Wozniak, K., \& Gillum, A. (2006). Imagination and false memory inductions: Investigating the role of process, content, and source of imaginations. Applied Cognitive Psychology, 20, 1119-1141. http://dx.doi.org/10.1002/acp.1265

Foley, M.A. (2012). Imagery encoding and false recognition errors: exploring boundary conditions of imagery is enhancing effects. Memory, 20(7), 700-716. http://dx.doi.org/10.1080/09658211.2012.697172 
Goff, L. M., \& Roediger, H. L. (1998). Imagination inflation for action events: repeated imaginings lead to illusory recollections. Memory and Cognition, 26, 20-33. http://dx.doi.org/10.3758/BF03211367

Gunter, R. W., Bodner, G. E., \& Azad, T. (2007). Generation and mnemonic encoding induce a mirror effect in the DRM paradigm. Memory \& Cognition, 35(5), 1083-1092. https://doi.org/10.3758/BF03193480

Israel, L., \& Schacter, D. L. (1997). Pictorial encoding reduces false recognition of semantic associates. Psychonomic Bulletin and Review, 4, 577-581.

http://dx.doi.org/10.3758/BF03214352

Johnson, M. K, \& Raye, C. L. (2000). Johnson, M. K., \& Raye, C. L. (2000). Cognitive and brain mechanisms of false memories and beliefs. In D. L. Schacter \& E. Scarry (Eds.), Memory, brain, and belief (p. 35-86). Harvard University Press.

Liddell, T. M., \& Kruschke, J. K. (2018). Analyzing ordinal data with metric models: What could possibly go wrong? Journal of Experimental Social Psychology, 79, 328-348. https://doi.org/10.1016/j.jesp.2018.08.009

Loftus, E. F. Levidow, B., \& Duensing, S. (1992). Who remembers best? Individual differences in memory for events that occurred in a science museum. Applied Cognitive Psychology, 6, 93-107. http://dx.doi.org/10.1002/acp.2350060202

Loftus, E. F., Miller, D. G., \& Burns, H. J. (1978). Semantic integration of verbal information into a visual memory. Journal of Experimental Psychology: Human Learning and Memory, 4(1), 19-31. https://doi.org/10.1037/0278-7393.4.1.19

Loftus, E. F., \& Palmer, J. C. (1974). Reconstruction of automobile destruction: An example of the interaction between language and memory. Journal of Verbal Learning \& Verbal Behavior, 13(5), 585-589. https://doi.org/10.1016/S0022-5371(74)80011-3 
Mahé, A., Corson, Y., Verrier, N., \& Payoux, M. (2015). Misinformation effect and centrality. European Review of Applied Psychology, 65(3), 155-162. https://doi.org/10.1016/j.erap.2015.03.001

Marks, D. (1973). Visual imagery differences in the recall of pictures. British Journal of Psychology, 64 (1), 17-24.

Mazzoni, G., \& Memon, A. (2003). Imagination can create false autobiographical memories. Psychological Science, 14(2), 186-188. http://dx.doi.org/10.1046/j.14321327.2000.01821.x

McDermott, K. B., \& Watson, J. M. (2001). The rise and fall of false recall: the impact of presentation duration. Journal of Memory and Language, 45, 160-176. http://dx.doi.org/10.1006/jmla.2000.2771

Monds, L. A., Paterson, H. M., \& Kemp, R. I. (2017). Do emotional stimuli enhance or impede recall relative to neutral stimuli? An investigation of two "false memory" tasks. Memory (Hove, England), 25(8), 945-952. https://doi.org/10.1080/09658211.2016.1237653

Nichols, R. M. \& Loftus, E. F. (2019). Who is susceptible in three false memory tasks? Memory, 27(7), 962-984. https://doi.org/10.1080/09658211.2019.1611862

Norman, K. A., \& Schacter, D. L. (1997). False recognition in younger and older adults: Exploring the characteristics of illusory memories. Memory \& Cognition, 25(6), 838-848. https://doi.org/10.3758/BF03211328

Olszewska, J., \& Ulatowska, J. (2013). Encoding strategy affects false recall and recognition: Evidence from categorical study material. Advances in Cognitive Psychology, 9(1), 44-52. https://doi.org/10.5709/acp-0130-0

Ost, J., Blank, H., Davies, J., Jones, G., Lambert, K., \& Salmon, K. (2013). False Memory $\neq$ False Memory: DRM Errors Are Unrelated to the Misinformation Effect. PLoS ONE, 8(4), 
e57939. https://doi.org/10.1371/journal.pone.0057939

Paivio, A. (2007). Mind and its evolution: a dual coding theoretical approach. Mahwah, NJ: Erlbaum Associates.

Paivio, A. (1991). Dual coding theory: retrospect and current status. Canadian Journal of Psychology, 45(3), 255-287.

Paivio, A. (1986). Mental representations: a dual coding approach. New York: Oxford University Press.

Paivio, A. (1971). Imagery and verbal processes. New York: Holt, Rinehart \& Winston.

Patihis, L., Frenda, S. J., \& Loftus, E. F. (2018). False memory tasks do not reliably predict other false memories. Psychology of Consciousness: Theory, Research, and Practice, 5(2), 140-160. https://doi.org/10.1037/cns0000147

Pérez-Mata, M. N., Read J. D., \& Diges, M. (2002). Effects of divided attention and word concreteness on correct recall and false memory reports, Memory, 10(3), 161-177. https://doi.org/10.1080/09658210143000308

Perrin, J., A Rachedi, A., \& C Gavras, C. (1969). Z [Motion picture]. France, Algérie: Cinema V

Pezdek, K., \& Lam, S. (2007). What research paradigms have cognitive psychologists used to study "False memory," and what are the implications of these choices? Consciousness and Cognition, 16(1), 2-17. https://doi.org/10.1016/j.concog.2005.06.006

Qin, J., Ogle, C. M., \& Goodman, G. S. (2008). Adults' memories of childhood: True and false reports. Journal of Experimental Psychology: Applied, 14(4), 373-391. https://doi.org/10.1037/a0014309

Robin, F. (2010). Imagery and memory illusions. Phenomenology and Cognitive Science, 9, 253-262. http://dx.doi.org/10.1007/s11097-010-9159-x 
Robin, F. (2011). Imagination and False Memories. Imagination, Cognition and Personality, 30(4), 407-424. http://dx.doi.org/10.2190/IC.30.4.e

Robin, F. \& Mahé, A. (2015). Effects of Image and Verbal Generation on False Memory. Imagination, Cognition and Personality, 35(1), 26-46. https://doi.org/10.1177/0276236615574488

Robin, F., Ménétrier, E., \& El Haj, M. (2019). How does imagery encoding impact false DRM recognition? The 13th Meeting of the Society for Applied Research in Memory \& Cognition (SARMAC XIII), Paper session: Memory Distortion I, June 6 - 9, 2019, Cape Cod, Massachusetts, USA.

Roediger, H. L., Balota, D. A., \& Watson, J. M. (2001). Spreading activation and arousal of false memories. In H. L. Roediger, J. S. Nairne, I. Neath, \& A. M. Surprenant (Eds.), The nature of remembering: Essays in honor of Robert G. Crowder (pp. 95-115). Washington, DC: American Psychological Association. http://dx.doi.org/10.1037/10394-006

Roediger, H. L., III, \& McDermott, K. B. (1995). Creating false memories: Remembering words not presented in lists. Journal of Experimental Psychology: Learning, Memory \& Cognition, 21, 803-814. http://dx.doi.org/10.1037/0278-7393.21.4.803

Roediger, H. L., Watson, J. M., McDermott, K. B., \& Gallo, D. A. (2001). Factors that determine false recall: A multiple regression analysis. Psychonomic Bulletin \& Review, 8(3), 385-407. https://doi.org/10.3758/BF03196177

Schacter, D. L., Israel, L., \& Racine, C. (1999). Suppressing false recognition in younger and older adults: The distinctiveness heuristic. Journal of Memory and Language, 40, 1-24. http://dx.doi.org/10.1006/jmla.1998.2611

Stadler, M. A., Roediger, H. L., \& McDermott, K. B. (1999a). Norms for word lists that create false memories. Memory \& Cognition, 27(3), 494-500.

https://doi.org/10.3758/BF03211543 
Wilkinson, C., \& Hyman, I. E., Jr. (1998). Individual differences related to two types of memory errors: Word lists may not generalize to autobiographical memory. Applied Cognitive Psychology, 12(Spec Issue), S29-S46. https://doi.org/10.1002/(SICI)10990720(199812)12:7<S29::AID-ACP596>3.0.CO;2-R

Zhu, B., Chen, C., Loftus, E. F., Lin, C., \& Dong, Q. (2013). The relationship between DRM and misinformation false memories. Memory \& Cognition, 41(6), 832-838.

https://doi.org/10.3758/s13421-013-0300-2 